\title{
Studies on Quality of Holes by using Micro-electro-discharge Machining Process
}

\author{
Zhang Liaoyuan $^{1, a}$, Ji Zhenming ${ }^{1, b}$, Lv Tingting ${ }^{1, c}$, Meng Fanjiang ${ }^{1, d}$ \\ ${ }^{1}$ Department of Electrical Engineering and Automation, Shenyang Ligong University, Shenyang, \\ 110159, China

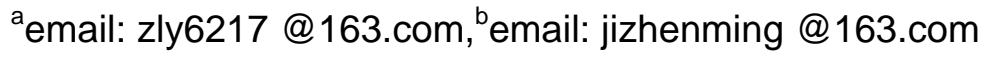

Keywords: Electric Parameters; Machining Accuracy; Spiral Drilling

\begin{abstract}
Based on the extensive application of small hole, how to guarantee the machining efficiency and machining quality of the hole at the same time is the key problem we need to solve. We analyze several factors affecting the machining quality of the hole and find their influence law on the machining accuracy of the hole. By comparing two kinds of EDM drilling methods, the method that makes the electrode wire spiral movement is more suitable for the machining of drilling and improves the hole accuracy and processing efficiency. These studies provide design experience for hole machining process.
\end{abstract}

\section{Introduction}

The head of rocket engine has many holes [1]. These holes are difficult to manufacture in the traditional way. The electric discharge machining draws our attention because of its simple equipment and high precision. So the study on the precision of small holes with micro EDM has important significance [2].

\section{Analysis of influence factors about the accuracy of holes}

As shown below, automatic feed device makes workpiece and electrode maintain a small discharge gap between them. When the pulse voltage is applied to the electrode and the workpiece, the instantaneous high temperature produces between workpiece and electrode. It removes metal from the workpiece and electrode.

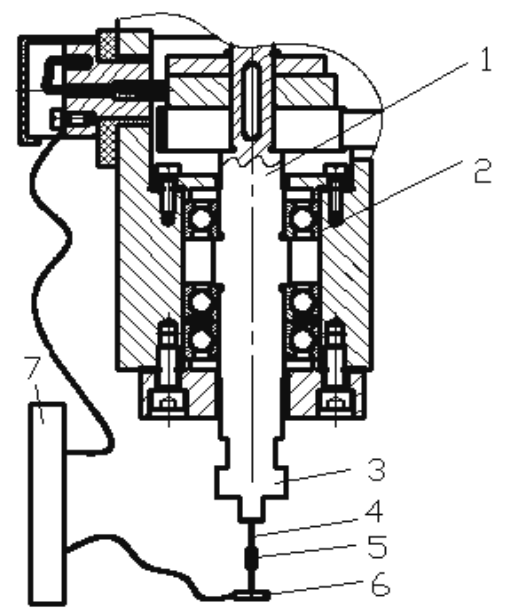

Fig.1. Micro EDM machining system

1-spindle 2- bearing 3-electrode holder 4-electrode 5-guide 6-workpiece 7-pulsed power source

From the Fig.1, we learn that there are two possible factors which will affect the precision of holes. The one is the precision of the device, and the other is the parameters of pulsed power source.

The error of the spindle and bearings leads to the existence of error of the spindle. This will make electrode swing. Electrode does relative movement around the ideal spindle axis and we put it into three separate forms of exercise [3]: pure axial moving, pure radial pulsation, and pure angle oscillation. Pure radial pulsation will make the size of holes increase and make the consistency of 
the size of holes difficult to guarantee. Pure angle oscillation will make the discharge uneven and holes' circularity is difficult to guarantee. So we add a guide sleeve. The structure of the guide sleeve is shown below:

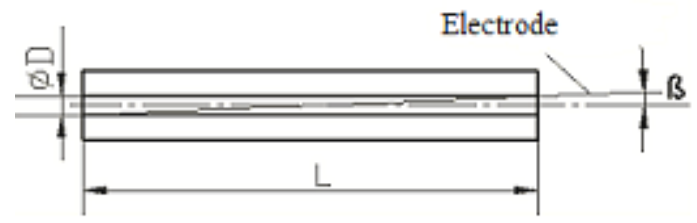

Fig.2. Guide sleeve

If the length of the guide sleeve is $\mathrm{L}=20 \mathrm{~mm}$, the inner diameter of holes is $\Phi \mathrm{D}=0.15^{+}{ }_{0}^{0.003} \mathrm{~mm}$ and the diameter of electrodes is $\Phi \mathrm{d}_{1}=\Phi 0.15^{0}{ }_{-0.002} \mathrm{~mm}$, then the largest gap between electrode and guide sleeve is: $\Delta=\Delta_{1}+\Delta_{2}=0.003 \mathrm{~mm}+0.002 \mathrm{~mm}=0.005 \mathrm{~mm}$. The angle between the guide sleeve axis and the electrode axis is $\beta=\arctan (\Delta /)=\arctan (0.005 \mathrm{~mm} / 20 \mathrm{~mm}) \approx 0.01432^{\circ}$. The distance of the lower end of guide sleeve to workpiece is $h=1 \mathrm{~mm}$. The axis of guide sleeve and the axis of the electrode intersect and the point of intersection is in the middle of the guide sleeve. The distance of the intersection to the bottom of guide sleeve is $l=L / 2=10 \mathrm{~mm}$. When the depth of holes is $\mathrm{H}=0.75 \mathrm{~mm}$, the error of holes caused by the gap between the guide sleeve and the electrode is $\Delta_{4}$ $=(\mathrm{H}+\mathrm{h}+1) * 2 * \tan \beta=(0.75 \mathrm{~mm}+1 \mathrm{~mm}+10 \mathrm{~mm}) * 2 * \tan 0.01432^{\circ} \approx 0.00587 \mathrm{~mm}$. So we can see that the bigger the depth of holes is, the greater the error of the diameter is. If the gap is too large, it will cause the uneven swing of the electrode and the roundness of holes is difficult to guarantee.

In Fig.3, when the axis of the electrode and the guide sleeve overlap, the electrode will swing slightly because of the spindle error and the gap. When it doesn't overlap, the electrode will swing heavily. In this case, the electrode axis deviates from the center line of machine tool spindle, which will lead to larger centrifugal force. So the electrode will swing more heavily and the roundness error of the hole increases. Therefore, we should make the center lines of the guide sleeve and the machine tool spindle overlapping. In addition, the center axis of guide sleeve should be vertical with the machine tool's worktable. Otherwise we will get inclined holes shown in fig.4.
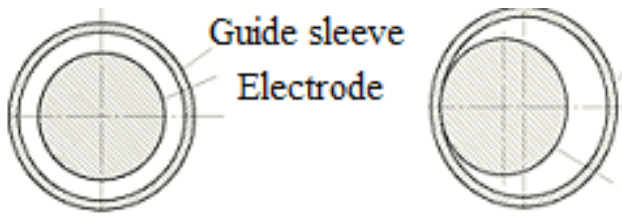

Guide sleeve

Electrode

Fig.3. Relative position of the guide sleeve and the electrode

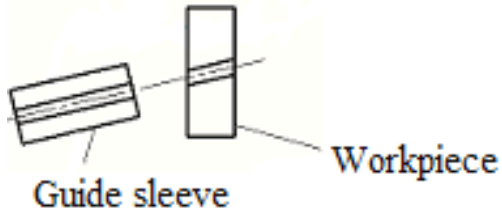

Fig.4. Inclined hole

In addition, because of the roundness error of electrode, holes' roundness error is bigger when the electrode does not rotate. So we should make spindle rotate. In addition, when we uses crooked electrode, the diameter of hole will be larger.

\section{Process experiment}

Equipment: SX-100HPM micro EDM CNC machine tool; the workpiece: the thickness is 2mm and the material is 1CrN9Ti; the fluid: EDM oil; spindle speed: 700rpm; electrode: copper electrode (hollow), the diameter is $\Phi 0.34^{0}{ }_{-0.002} \mathrm{~mm}$; guide sleeve: the length is $20 \mathrm{~mm}$, inner diameter size is $\Phi 0.34^{+}{ }_{0}^{0.003} \mathrm{~mm}$ and the distance of the lower end of the guide sleeve to the workpiece is $1 \mathrm{~mm}$; flushing way: side flushing + electrode internal flushing.

Set pulse voltage 110V, pulse width 5us and pulse frequency $100 \mathrm{Khz}$.

Table 1 The holes' diameter and cylindricity change along with the peak current

\begin{tabular}{ccccccccc}
\hline variable & \multicolumn{10}{c}{ data } \\
\hline the current /A & 20 & 28 & 36 & 44 & 52 & 60 & 68 & 76 \\
aperture $/ \mathrm{mm}$ & 0.375 & 0.377 & 0.380 & 0.382 & 0.382 & 0.384 & 0.385 & 0.385 \\
cylindricity/mm & 0.0052 & 0.0050. & 0.0049 & 0.0047 & 0.0042 & 0.0046 & 0.0049 & 0.0052 \\
\hline
\end{tabular}

The holes' size becomes larger when the current becomes greater. The reason is that the current increases, leading to the increase of the energy of single pulse and discharge gap. With the increase of current changing in the range of 20 52A, cylindricity gradually decreases. The reason is that the 
ratio of pulse width and pulse interval is larger. The effective discharge time is longer, so the discharge energy is larger [4]. In a short time, it produces great explosive force so that electric corrosion product can be discharged quickly. With the increase of current changing in the range of 52 76A, cylindricity becomes bigger. The reason is that the peak current increases, instantaneous energy increases and electric corrosion product increases [5]. It is difficult to discharge electric corrosion products and then exacerbates the secondary discharge phenomenon. Then the taper of holes increases, the cylindricity error increases.

Set the pulse voltage $110 \mathrm{v}$, the peak current $20 \mathrm{~A}$ and pulse frequency $100 \mathrm{Khz}$ Table 2 The holes' diameter and cylindricity change along with the pulse width

\begin{tabular}{cccccccc}
\hline variable & \multicolumn{7}{c}{ data } \\
\hline pulse width $/ \mu \mathrm{s}$ & 2 & 2.5 & 3 & 3.5 & 4 & 4.5 & 5 \\
aperture $/ \mathrm{mm}$ & 0.372 & 0.373 & 0.375 & 0.377 & 0.378 & 0.380 & 0.381 \\
cylindricity $/ \mathrm{mm}$ & 0.0066 & 0.0063 & 0.0060 & 0.0059 & 0.0056 & 0.0053 & 0.0051 \\
\hline
\end{tabular}

When the pulse width increases, the pore size increases. The reason is that when pulse width increases, the energy of single pulse increased, resulting in the increase of the discharge gap's size. In addition, with the increase of pulse width, cylindricity gradually decreases, but the change is not obvious. The reason is that when the pulse width increases, pulse energy increases to maintain a stable working condition.

Through the above data, it indicates that the influence of electrical parameters on the machining accuracy of holes is not obvious, so we put forward a new method and we design an experiment.

Experimental conditions: raw material: outer diameter: $\Phi 10 \mathrm{~mm}$, inner diameter: $\Phi \mathrm{D}=\Phi 6 \mathrm{~mm}$, the length: $140 \mathrm{~mm}$. The workpiece we need is in fig.4.

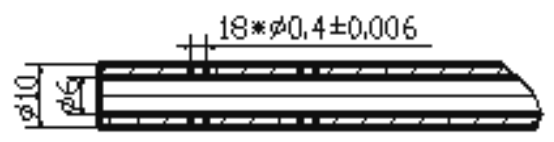

Fig. 5 The workpiece we need

The workpiece material: 1CrN9Ti. Spindle speed: 700rpm.Electrode: copper (hollow), size is $\Phi d_{1}=\Phi 0.3^{0}{ }_{-0.002} \mathrm{~mm}, \Phi d_{2}=\Phi 0.36^{0}{ }_{-0.002} \mathrm{~mm}$. Guide sleeve: the length $\mathrm{L}=20 \mathrm{~mm}$, inner diameter is $\Phi D_{1}=\Phi 0.3^{+}{ }_{0}^{0.003} \mathrm{~mm}, \Phi D_{2}=\Phi 0.36^{+}{ }_{0}^{0.003} \mathrm{~mm}$ and the distance of the lower end of guide sleeve to workpiece is $\mathrm{h}=1 \mathrm{~mm}$.Flushing: side flushing + electrode internal flushing. Electric parameters: voltage $110 \mathrm{~V}$, peak current 52A, pulse width 5us and pulse frequency $100 \mathrm{KHz}$.

Try to machine small holes in two different ways. In order to reduce the taper of the hole, feed depth is $\Phi D+H=8 \mathrm{~mm}$. $\Phi D$ is the inner diameter of the tube and $\mathrm{H}$ is the depth of hole.

(1) Use electrode with the diameter of $\Phi d_{2}=\Phi 0.36^{0}{ }_{-0.002} \mathrm{~mm}$ to feed directly

We get the hole with the diameter of $\Phi D_{3}=\Phi 0.4^{+}{ }_{0}^{0.01} \mathrm{~mm}$. The hole we get is in Fig.5. In order to improve the machining efficiency, we use the electric parameters higher. This will cause the increase of the pulse energy in unit time, but the electric corrosion product increases and the chip removal is poor. The secondary discharge increases, resulting in the increase of aperture. When holes are just drilled through, the electric corrosion product will flow out of the outlet of holes, which reduces the secondary discharge. This makes holes with taper.

(2) Use electrode with the diameter of $\Phi d_{1}=\Phi 0.3^{0}{ }_{-0.002} \mathrm{~mm}$ to feed in spiral way We get holes with diameter of $\Phi \mathrm{D}_{4}=\Phi 0.4^{+}{ }_{0}{ }^{0.006} \mathrm{~mm}$. The holes we get are in Fig.6.

\section{Figure 6 The hole without taper}

By programming, make the trajectory of the bottom of the electrode similar to a spring. The radius $\mathrm{R}=0.027 \mathrm{~mm}$, pitch $\mathrm{S}=0.01 \mathrm{~mm}$. The center line of the electrodes does rotary movement around the axis of the aperture and the velocity is $\mathrm{V}=0.25 \mathrm{~mm} / \mathrm{s}$. The pitch value is reasonable. If the pitch value is too small, the processing efficiency is too low. If the pitch value is too big, the chip removal and deionization is poor and it is easy to produce short circuit. Processing efficiency would decline and it will reduce the machining precision. Linear velocity $\mathrm{V}$ is obtained through experiments. When linear velocity is too large, the bottom of the electrode will swing too heavily, 
which makes the aperture at the exit of the hole greater than the aperture at the entrance of the hole. When the linear velocity is too small, the bottom of the electrode will swing too slightly. So the swing of the electrode can't compensate the taper of the hole, which makes holes with taper.

The dimension tolerance of electrode is $\Delta_{1}=0.002 \mathrm{~mm}$. The dimension tolerance of the inner aperture of the guide sleeve is $\triangle_{2}=0.003 \mathrm{~mm}$. The largest gap between the electrode and the guide sleeve is $\triangle=\Delta_{1}+\triangle_{2}=0.002 \mathrm{~mm}+0.003 \mathrm{~mm}=0.005 \mathrm{~mm}$. The error caused by the gap is $\Delta_{3}=(\mathrm{H}+\mathrm{h}$ $+1) * 2 * \tan \beta=(2 \mathrm{~mm}+1 \mathrm{~mm}+10 \mathrm{~mm}) * 2 * \tan 0.01432^{\circ} \approx 0.00649 \mathrm{~mm}$. The enlargement of the diameter of holes caused by other factors is $\Phi D_{4}-\Phi d_{1}-R^{*} 2-\triangle_{3}=\Phi 0.4 \mathrm{~mm}-\Phi 0.3 \mathrm{~mm}$ $-0.027 \mathrm{~mm} * 2-0.00649 \mathrm{~mm}=0.03951 \mathrm{~mm}$. We use the electrode with the same diameter to machine holes in the first method and holes' size we get is $\Phi D_{5}=\Phi 0.34+00.013 \mathrm{~mm}$. Similarly, the enlargement of the diameter of holes caused by other factors is $\Phi \mathrm{D}_{5}-\Phi \mathrm{d}_{1}-\triangle_{3}=0.34 \mathrm{~mm}-0.3 \mathrm{~mm}-0.00649 \mathrm{~mm}=0.03351 \mathrm{~mm}$ and the value is less than $0.03951 \mathrm{~mm}$. The difference of the both is $0.03951 \mathrm{~mm}-0.03351 \mathrm{~mm}=0.006 \mathrm{~mm}$. This difference is caused by the increased centrifugal force. When we use the second way, the center line of the electrodes does rotary movement around the center line of the aperture, which makes the centrifugal force increase, reducing the taper of the hole. In addition, the precision of holes is higher in this way. The reason is that the center line of the electrode and the center line of the hole don't overlap, which causes larger gap to accommodate electric corrosion products. At the same time, the rotating and moving of the electrode makes the electric corrosion product quickly go into the gap, which is conducive to exhaust discharge product out of the gap. It greatly reduces the secondary discharge and short circuit, which greatly improves the utilization rate of pulse, so that the machining efficiency is improved obviously and the difference of the diameter of holes decreases.

\section{Conclusion}

(1) The precision of mechanical system is the main factor affecting the accuracy of holes, such as the straightness of the electrode and the precision of internal diameter of the guide sleeve.

(2) The holes' size increases when the pulse width, peak current and the pulse frequency increase. The cylindricity of holes decreases with the increase of pulse width and pulse frequency. With the increase of the peak current, the cylindricity error decreases first and then increases. But the impact on the cylindricity error caused by the electrical parameters is not very obvious.

(3) We put forward a new method that makes the electrode feed in a spiral way continuously. The method makes the processing efficiency improved and the cylindricity error decreases.

\section{References}

[1] Y.H. Guu, Max Ti-Kuang Hou. Effect of parameters on surface textures in EDM of alloy [J].Materials Science and Engineering A 2007, 466: 61-67.

[2] Konig W, Panten U.Effective machining process even for ceramic [C].Proceedings of the International Symposium of Electro-machining, ISEM-X, Magdeburg,1992:269-286

[3] Guo Hongqin, Pei Jingyu, Li Wuyi. The process study on the machining of holes using micro electrical discharge[J]. aerospace manufacturing technology, 2005,05:19-21+29.

[4] Cui Jingzhi. The basic law of micro EDM and its simulation study [D]. Harbin Institute of Technology, 2007

[5] An Libao. Electric spark precision machining and gap characteristics research [J]. aviation precision manufacturing technology, 1994,06:16-18. 